\title{
Investigating the effect of taper length on sensitivity of the tapered-fiber based temperature sensor
}

\begin{abstract}
A temperature sensor using single-mode tapered fiber is presented. To better understand the behaviour of a tapered optical fiber, transmission experiments with different taper profiles, specifically waist length were performed. The effects of taper profiles on the sensitivity of the sensor were also investigated. It is demonstrated that careful selection of the taper profile can increase the sensitivity of the sensor. In our experiment, a good temperature sensing result was achieved using the optimum parameter. The best sensitivity achieved was $45.5 \mathrm{pm} /{ }^{\circ} \mathrm{C}$ that measured the range of temperature from $30^{\circ} \mathrm{C}$ to $120^{\circ} \mathrm{C}$. The fabricated sensors are easy to fabricate and relatively low cost. Our results indicate that the tapered fiber based temperature sensor has high sensitivity and good repeatability.
\end{abstract}

Keyword: Fiber optic sensor; Tapered fiber; Temperature sensor 